\title{
EVALUATION OF OPERATIONAL EFFICIENCY OF URBAN ROAD NETWORK USING TRAVEL TIME RELIABILITY MEASURES
}

\author{
Sathya Prabha $\mathbf{R}^{1}$, Samson Mathew ${ }^{2}$ \\ ${ }^{1}$ Post Graduate, Student, Transportation Engineering and Management, ${ }^{2}$ Professor, Department of Civil Engineering, \\ National Institute of Technology, Tiruchirappalli \\ sathyaprabhacivil@gmail.com,sams@nitt.edu
}

\begin{abstract}
Efficiency of the road network system is analyzed by travel time reliability measures. The study overlooks on an important measure of travel time reliability and prioritizing Tiruchirappalli road network. Traffic volume and travel time were collected using license plate matching method. Travel time measures were estimated from average travel time and 95th travel time. Effect of non-motorized vehicle on efficiency of road system was evaluated. Relation between buffer time index and traffic volume was created. Travel time model has been developed and travel time measure was validated. Then service quality of road sections in network were graded based on travel time reliability measures.
\end{abstract}

Keywords: Buffer Time Index (BTI); Average Travel Time (ATT); Travel Time Reliability (TTR); Buffer Time (BT).

\section{INTRODUCTION}

Congestion, delay and unreliability are terms that are most associated with present-day travel. Both individuals and companies suffer from economic losses due to lost time as well as additional vehicle operating and detour costs. Travel time has been a critical measure and important measure used to evaluate an effectiveness of the road. For this reason, travel time reliability measure is important to travelers in order for them to plan their trips effectively as well as shippers for them to plan and select routes appropriately.

This paper will describe how measures of travel time reliability are used in that evaluation and better understanding of the transportation system. It will explore ways in which travel time reliability is measured using archived data over a segment. It is important to communicate the ways that transportation investments can benefit an entire region. In this study, a methodology is presented to estimate travel time reliability based on modeling travel time considering travel time reliability and safety for evaluation of operational efficiency of road. Travel time is modeled as a function of traffic volume, speed and distance.

\section{BACKGROUND}

\subsection{Methods of Estimating Travel Time}

Travel time evaluation methods consist of direct measurements and estimations. The most popular method to measure travel time directly has been the floating car technique. A GPS device to record the vehicle trajectory along with time stamps. Another simple technology is Video
Image Processing (VIP) system. In this method video cameras are placed at origin and destination of each sections of the road system. Travel time of vehicles can be directly obtained by synchronizing videos which were placed at the ends of the segments.

\subsection{Definitions of Travel Time Reliability}

Ebeling (1997) defined reliability as "the probability that a component or system will perform a required function for a given period of time when used under stated operating conditions. It is the probability of a non-failure over time." This definition of reliability is widely accepted in engineering. In the 1998 California Transportation Plan, reliability is defined as the level of variability between the expected travel time and the actual travel time experienced. In that definition, the expected travel time is based on scheduled or average travel time, while the actual travel time incorporates the effects of non-recurrent congestion. The difference between the two values may be small, labeling the facility as "reliable", when it is consistently congested.

\subsection{Measures of Travel Time Reliability}

Measures of travel time reliability attempt to quantify both the variability in travel times across different days and months. Travel time reliability is an alternative to the volume-tocapacity ratio, and can greatly help to determine the adequacy of the service provided by a transportation network. Whereas the V/C ratio focuses specifically on the need to design for a capacity that will meet the demand on a roadway, and therefore tends to lead to recommendations to build more roadways, travel time reliability measures are 
oriented towards better management of the existing system. The most common measures include various indices suggested by the FHWA (2005). Indices of reliability are commonly divided into statistical and buffer measures. Statistical methods include travel time window and percent variation shown in equations. Buffer measures are,

$90^{\text {th }}$ or $95^{\text {th }}$ Percentile Travel Time: The 95th percentile travel time ensures the user is only late 1 out of every 20 trips.

Travel Time Index: the average amount of time it takes to travel during peak hours compared to free flow conditions computed as average travel time divided by free flow travel time.

Buffer Index: the extra time that Travelers add to travel to make sure they are on time most of the time.

$$
\text { buffer index }=\frac{95 \text { th percentile travel time }- \text { ATT }}{\text { ATT }}
$$

Planning Time Index: the total time needed to plan for an ontime arrival $95 \%$ of the time, computed as $95^{\text {th }}$ percentile travel time divided by free-flow travel time.

\section{METHODOLOGY}

Urban road network have been taken for the evaluation of efficiency. Urban road network from Tiruchirappalli district through Bharadidhasan road, Puthur EVR road, Thennur high road and Thillainagar main road. Full observation was made about Tiruchirappalli urban areas. Video graphic survey was conducted from 8:00 AM to 10:00 AM for various selected roads in both directions for different day. By synchronizing videos, traffic volume and travel time was obtained which were the data to estimate the travel time reliability measures. 95th percentile travel time and average travel time obtained from cumulative percentage of frequency and arithmetic mean equation (Traffic Engineering, Kadiyali).

Buffer time index was estimated and operational efficiency of each road was evaluated from above parameters. Impact of Non-Motorized Vehicle (NMV) on buffer time index is to be analyzed. After detailed study was carried out about factors that influence the travel time and operational efficiency of roads, suitable parameters were considered to develop the travel time model. Then developed model was validated by data obtained from traffic survey.

\section{TRAVEL TIME ANALYSIS}

Traffic volume studies were conducted at a given location. Traffic volume count analysis gives a measure of composition of heterogeneous traffic on the selected road network. From Figure $4.1-4.8$ gives average travel time for NMV is comparatively high.

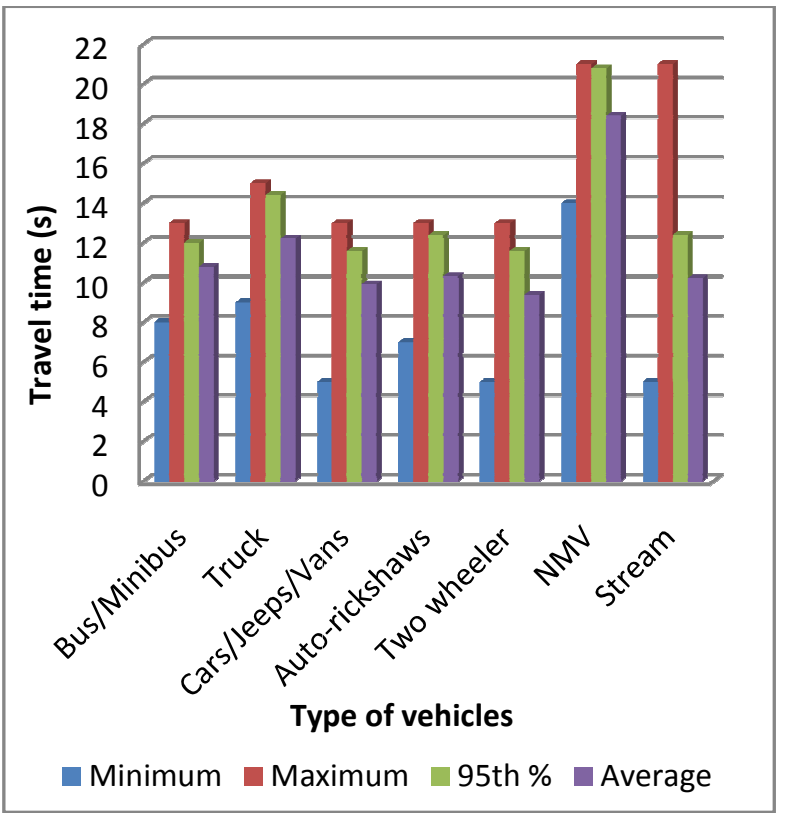

Figure 4.1 Bharathidasan road: Court to American hospital

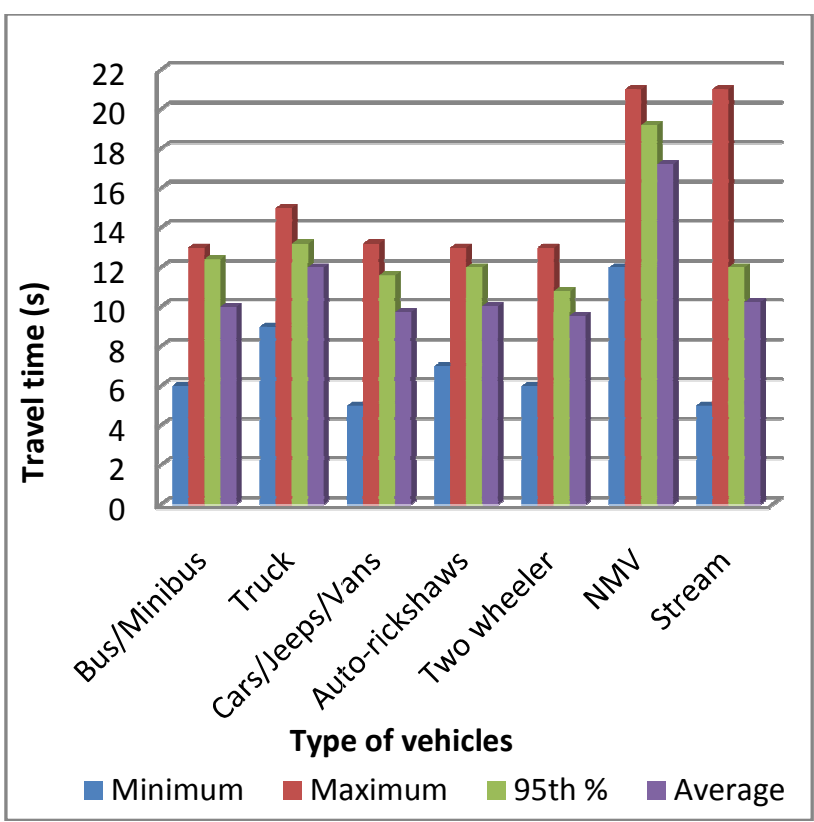

Figure 4.2 Bharathidasan road: American hospital to Court 


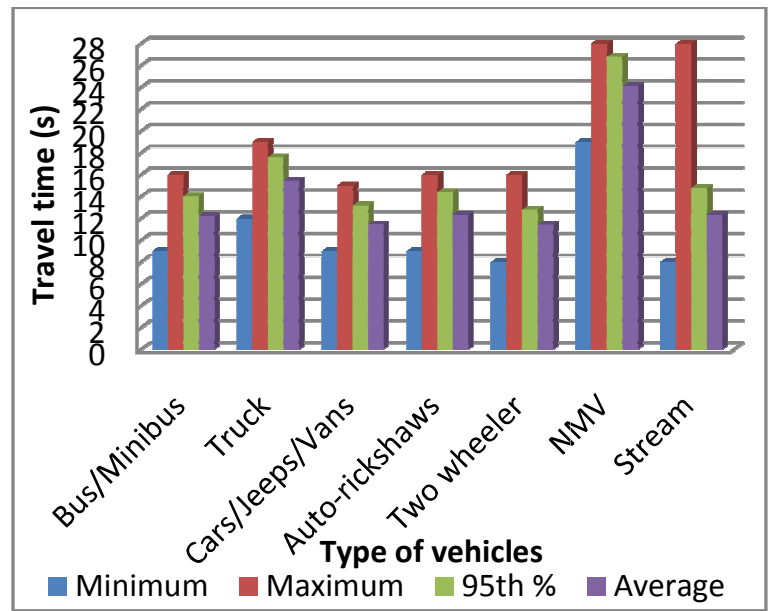

Figure 4.3 Puthur EVR road: Puthur junction to MGR statue

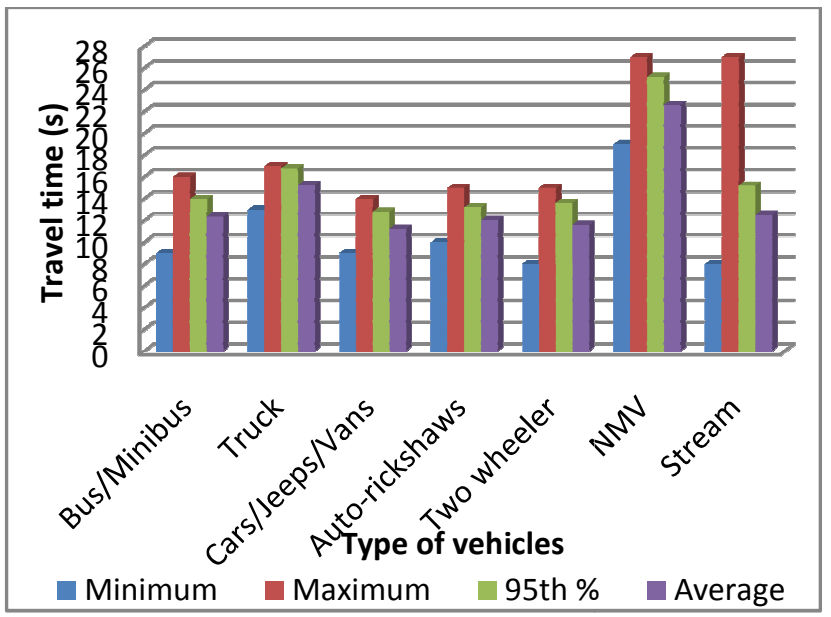

Figure 4.4 Puthur EVR road: MGR statue to Puthur junction

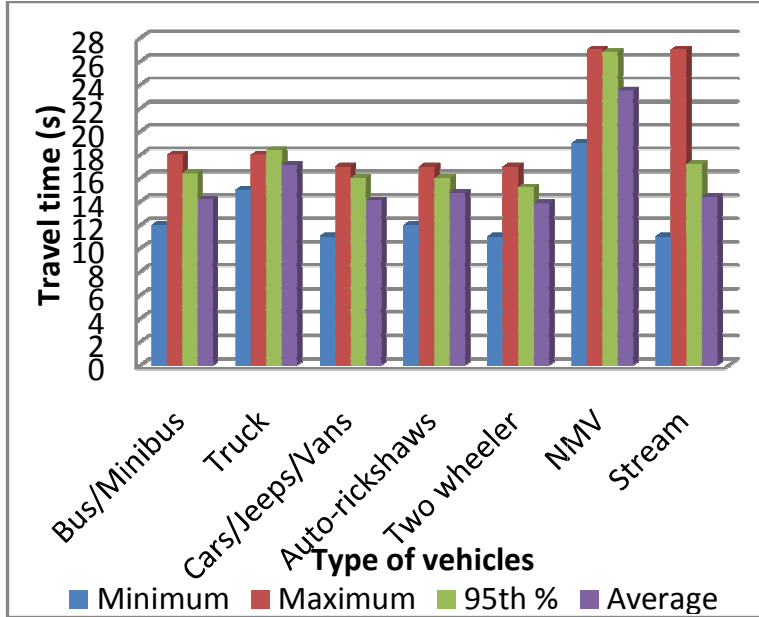

Figure 4.5 Thennur high road: Puthur junction to Thennur

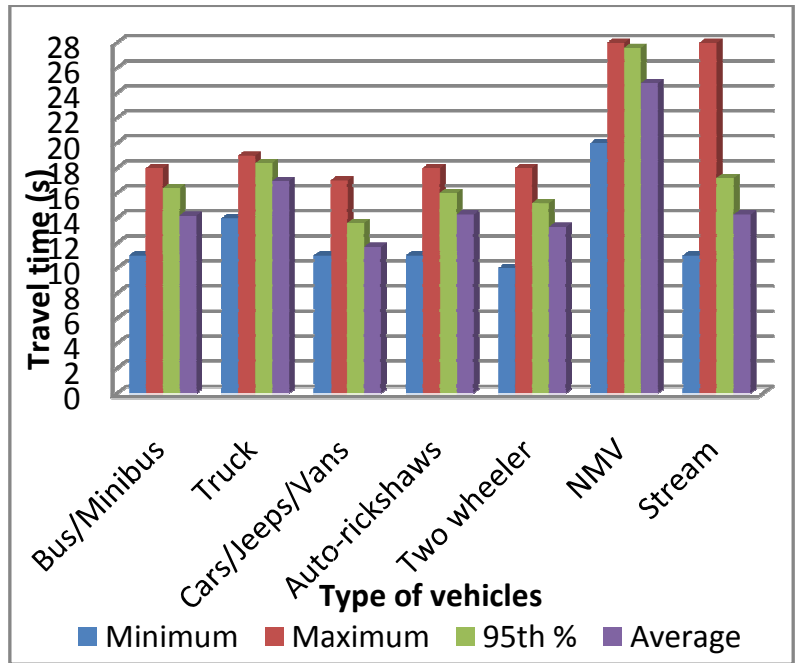

Figure 4.6 Thennur high road: Thennur to Puthur junction

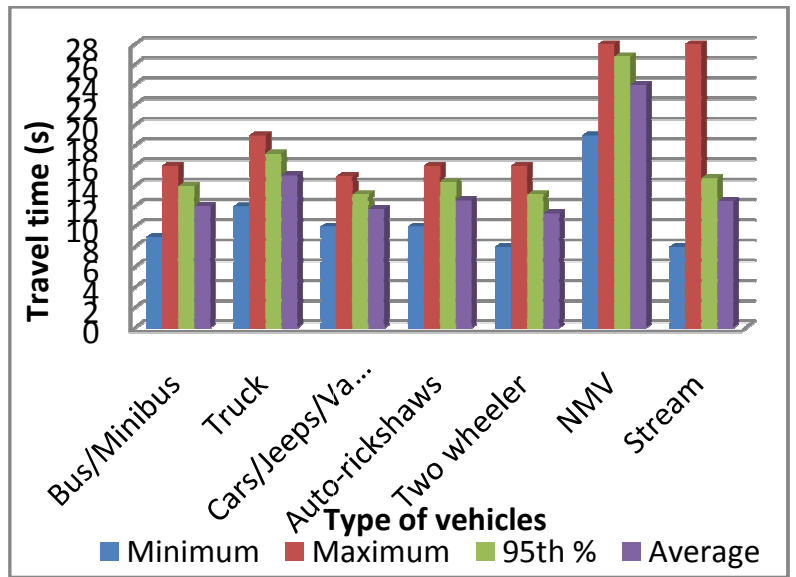

Figure 4.7 Thillainagar main: Salai junction to Thennur

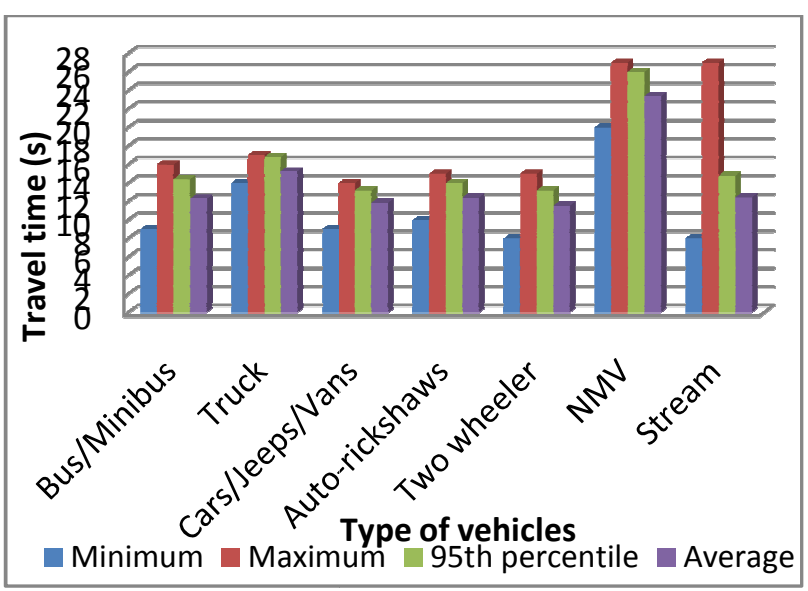

Figure 4.8 Thillainagar main: Thennur to Salai junction 
Truck has second highest average travel time. Thennur high road has the highest and Bharathidasan road has least average travel time and has 95th percentile travel time when compare to other roads. When considering direction wise, Bharathidasan road has same and less average travel time in both directions. And Thennur high road has a large average travel time in direction of Puthur junction to Thennur junction. Bharathidasan road has less 95 th percentile travel time.

\section{BUFFER MEASURE}

Based on BTI, Bharathidasan road was graded as having more efficiency because it has low BTI and Thennur high road has least efficiency because it has more BTI. Thillainagarmain road has second highest efficiency and Puthur EVR road has third highest efficiency. Impact of nonmotorized vehicle high for Puthur EVR road. Then impact of
NMV on BTI was reduced inorder of Thillainagarmain road, Thennur high road and Bharathidasan road.

Study were carried out for another network selected from Salai Road in Tiruchirappalli as following links NachiyarKovil - Thillainager Road Junction, Thillainager Road Junction - Sastri Road Junction and Sastri Road Junction - Banana leaf. Analysis was carried out for different type of vehicles in both directions. From the analysis, based onBTI, Sastri road Junction - Banana Leaf road section was graded as having more efficiency.NachiyarKovil - Thilainagar Road Junction road has second highest efficiency and Thilainagar Road Junction - Sastri road Junction has third highest efficiency.

\subsection{Relation between buffer time index and traffic flow}

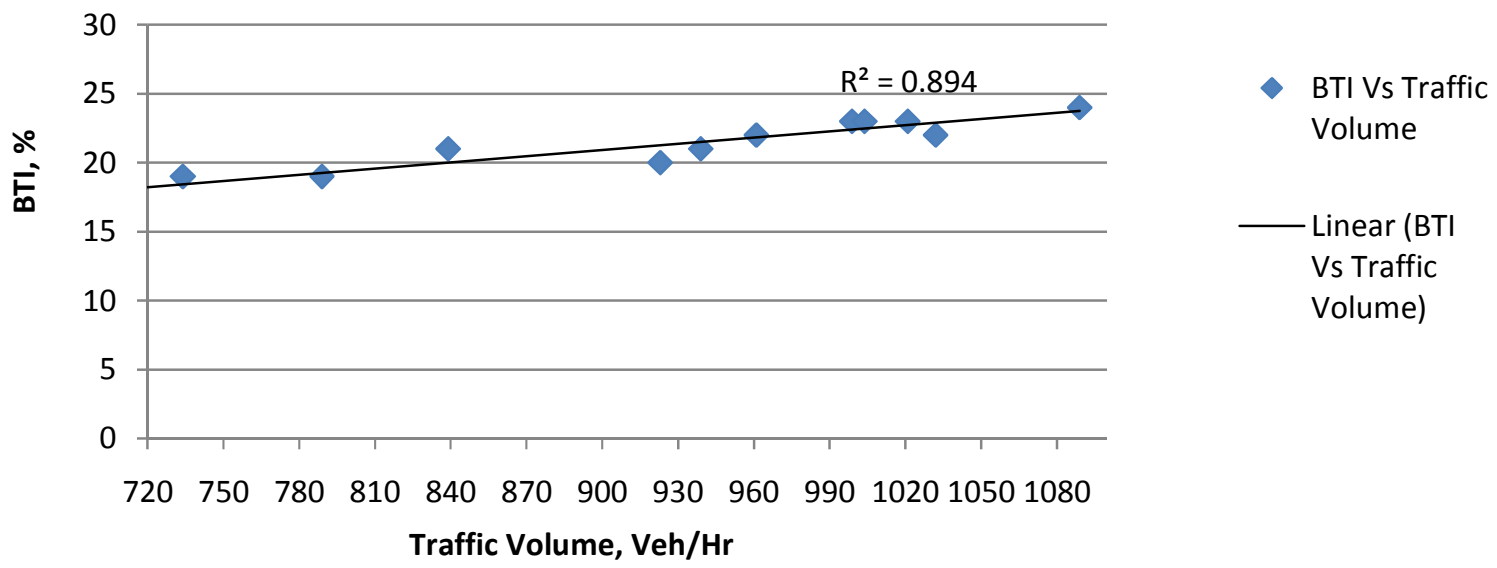

Figure 5.1 relations between BTI and traffic flow

Figure 5.1 shows relation between buffer time index and traffic flow which has been developed using data obtained. The observations made for each link in every direction for one hour traffic flow. From this relation, buffer time index increases with traffic volume. In this study the adjusted Rsquared statistic is 0.895 for this relationship. So this relation is in good fit. From this model we can find required buffer time index for different traffic flow.

\section{TRAVEL TIME MODELING}

\subsection{Regression Analysis}

A regression model expresses a 'dependent' variable as a function of one or more 'independent' variables, generally in the form:

$$
y=\alpha+\beta_{1} x_{1}+\beta_{2} x_{2} \ldots+\epsilon
$$

The line that expresses the relationship between the $y$ variable, called the dependent variable. Regression with a single dependent variable $\mathrm{y}$ whose value is dependent upon the independent variable $x$ is expressed as

$$
y=\alpha+\beta x+\epsilon
$$

Where $\alpha$ is a constant, so is $\beta$. $x$ is the independent variable and $\epsilon$ is the error term. Standard error of the regression $\epsilon$ is considered normally distributed with a mean of zero, and a variance equal to $\sigma 2$. 


\subsection{Model Estimation}

Because travel time is a continuous variable, the first modeling formulation used was a linear regression with travel time as the dependent variable. Independent variables that were included in the model were average travel time, volume and distance travelled by vehicle. Linear regression analysis yielded reasonable parameter estimates and model fit.

Travel time $=50.970+\left(0.116^{*}\right.$ Length $)-(2.060 *$ Speed $)+$ $(0.022 *$ Volume $)$

Table 6.1 travel time model

\begin{tabular}{|l|l|l|l|l|l|}
\hline Parameters & Explanation & Coefficients & Standard Error & t Stat & P-value \\
\hline Constant & Intercept & 50.970 & 9.989 & 5.103 & 0.000 \\
\hline Length & Distance travelled by vehicle (m) & 0.116 & 0.141 & 0.820 & 0.000 \\
\hline Speed & $\begin{array}{l}\text { Speed during the 15-minute time } \\
\text { period (km/h) }\end{array}$ & -2.060 & 1.117 & -1.845 & 0.000 \\
\hline Volume & $\begin{array}{l}\text { Volume during the 15-minute time } \\
\text { period (PCU/h) }\end{array}$ & 0.022 & 0.102 & 0.218 & 0.035 \\
\hline $\begin{array}{l}0.997 \\
\text { R Square } \\
\text { Observations }\end{array}$ & \multicolumn{2}{|l}{} & & \\
\hline
\end{tabular}

In this model length gives positive effect to travel time of vehicle. The reason that if the length increases, travel time and overtaking factor of vehicle will affect. Finally an increase in the length of the segment will obviously increase the vehicle travel time. Speed of vehicle has negative influence. Speed can be considered the single most important factor influencing travel time. When speed increases, travel time of vehicle will reduce. Volume has positive influence on travel time model. Volume can have an additional impact on the flow. Increase in volume will obviously cause the congestion at the peak hour traffic flow. When congestion increases, travel time of vehicle will be increased.

In this study the adjusted R-squared statistic is 0.996 for the travel time model. In addition to evaluating the model fit, it is important to ensure that all the parameters included in the models are relevant. As shown in Tables 6.1, the t-statistics for all parameters are between -2.00 to 2.00 , making them significant at the $95 \%$ confidence level. $\mathrm{P}$-values are less than 0.05 , this make the model significant at the $95 \%$ confidence level.

Another way to assess the effectiveness of the model is by visual assessment of predicted and actual travel times. Figure 6.1displays actual and predicted average travel times for Bharathidasan road in the direction of Court to American hospital from 8.00 AM to $10.00 \mathrm{AM}$. It is evident from the graphs that the estimated model is effective at predicting the travel time on the selected road.

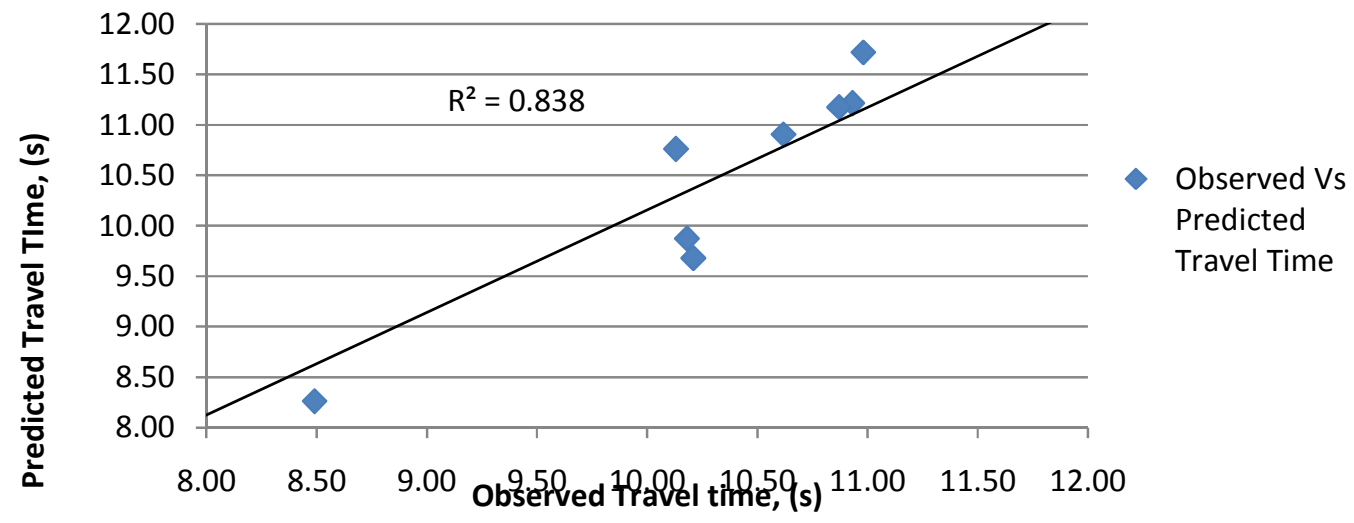

Figure 6.1 variation of travel time 


\section{SUMMARY \& CONCLUSIONS}

The prime focus of the study relies on use of travel time reliability measures to find the operational efficiency of urban road network in Tiruchirappalli city. Literature reviews were done to prioritize the techniques to be used to find the travel time of vehicle. Analysis of traffic parameters and traffic volume data were carried out on the selected roads. From these, buffer time index in selected road was evaluated for various types of vehicle, peak hour and in direction wise.

From the analysis, Bharathidasan road is the only six lane road in Tiruchirappalli city and has better maneuverability allowing the driver to move fast with less travel time. Thillai Nagar and Thennur High road area bounded by commercial centres carries large amount of traffic, because of that Thennur high road has high travel time. Puthur EVR road has high impact due to non-motorized vehicle, which reduced $24.35 \%$ of buffer time index compare to other selected roads. Next to Puthur EVR road, BTI reduced in order of Thillainagar main road, Thennur high road and Bharathidasan road.

Bharathidasan road has high efficiency due to less BTI, then efficiency decreased in order of Thillainagar main road, Puthur EVR road and Thennur high road. Considering Salai road network, based on buffer time index, Sastri road Junction - Banana Leaf road section was graded as having more efficiency because it has low buffer time. NachiyarKovil - Thilainagar Road Junction road has second highest efficiency and Thilainagar Road Junction - Sastri road Junction has third highest efficiency. Validation of travel time model was carried out by different ways like statistical method and visual graphical method. Finally model was proved as fit to find future variation in travel time of vehicle.

\section{REFERENCES}

[1] CRRI, "Evaluation of Operational Efficiency of Highway Network using Travel Time Reliability Measures",Annual Report, New Delhi, 2010.

[2] Elefteriadou.L, "A framework for defining and estimating travel time reliability",85th Annual Transportation Research Board Conference, Washington, DC, 2006.

[3] Federal Highway Administration, "Travel Time Reliability: Making It There On Time, All the Time", Texas transportation Institute with Cambridge Systematics Inc, 2006.

[4] Florida DOT (2007) Travel Time Reliability and Truck Level of Service on the Strategic Intermodal System.

[5] Hans van Lint and Henk van Zuylen,"The Effects of Traffic Accidents on Travel Time Reliability", 11th International IEEE Conference on Intelligent Transportation Systems, Beijing, Oct 12-15, 2008.

[6] He QiChao and Wang Luo,"Reliability Research on Travel Time of Transportation Network", International
Conference on Intelligent Computation Technology and Automation, 2010.

[7] Huizhao Tu and Hans van Lint,"Travel Time Variability versus Freeway Characteristics",IEEE Intelligent Transportation Systems Conference, Toronto, Canada, September 17-20, 2006.

[8] Jennifer Dill and Robert Bertini,"Travel Time Reliability in Regional Transportation Planning”,Field area paper, Portland State University, USA, 2007.

[9] Maria Martchouk and Fred L. Mannering, "Travel Time Reliability in Indiana",Joint Transportation Research Program , Final Report, FHWA, 2010.

[10] Ma Hongwei and Fu Liyou, "Study on The Reliability of Travel Time in Transportation Network", International Conference on Intelligent System Design and Engineering Application, 2012.

[11] Michael Mauch and PravinVaraiya,"Components of Congestion: Delay from Incidents, Special Events, Lane Closures, Weather, and Excess Demand",Transportation Research Board of the National Academies, Washington,D.C., pp. 84-91, 2006.

[12] PronyBonnaireFils, "Modeling Travel Time and Reliability on Urban Arterials for Recurrent Conditions",Graduate school thesis and dissertations. University of South Florida, 2012.

[13] Shawn M. Turner, William L. Eisele, Robert J. Benz, and Douglas J.Holdener,"Travel time data collection handbook". Federal Highway Administration, Washington, DC. FHWA-PL-98-035, 1998.

[14] XU Minghua and XU HaiwenAssessing Performance Reliability of Transportation Networks under Demand and Supply Uncertainties. China. 